\title{
Variabilidade espacial de Colletotrichum truncatum em campo de soja sob três níveis de sanidade de sementes
}

\author{
Rafael Noetzold ${ }^{1}$, Marcelo de Carvalho Alves², Daniel Cassetari Neto ${ }^{3}$, Andreia Quixabeira Machado ${ }^{4}$
}

\begin{abstract}
${ }^{1}$ Universidade Federal de Mato Grosso, Faculdade de Agronomia, Medicina Veterinária e Zootecnia, Programa de Pós-Graduação em Agricultura Tropical, Av. Fernando Correa da Costa, 2367, Boa Esperança, Cuiabá, MT, 78060-900, (UFMT); ${ }^{2}$ Universidade Federal de Lavras, Departamento de Engenharia, Lavras, MG; ${ }^{3}$ UFMT, Departamento de Fitotecnia e Fitossanidade; ${ }^{4}$ Centro Universitário - UNIVAG, Av. Dom Orlando Chaves, 2655, Várzea Grande, MT
\end{abstract}

Autor para correspondência: Rafael Noetzold (rafael_noetzold@hotmail.com)

Data de chegada: 08/08/2013. Aceito para publicação em: 03/02/2014.

1913

\section{RESUMO}

Noetzold, R.; Alves, M.C.; Cassetari Neto, D.; Machado, A.Q.. Variabilidade espacial de Colletotrichum truncatum em campo de soja sob três níveis de sanidade de sementes. Summa Phytopathologica, v.40, n.1, p.16-23, 2014.

Objetivou-se caracterizar a variabilidade espacial da incidência de Colletotrichum truncatum (Schwein) em sementes colhidas de soja e determinar o melhor modelo e método de semivariograma que represente a incidência de $C$. truncatum dessas sementes. O experimento foi realizado em condições de campo na safra 2009/10, em 3 parcelas de $9,9 \times 10 \mathrm{~m}$, com sementes inoculadas com C. truncatum. O inóculo correspondeu a 0,$8 ; 1,6$ e 2,4\% do total semeado. Foram demarcadas 3 malhas com receptores GNSS, totalizando 112 pontos em cada parcela distanciados a 1,3 m na linha. No final do ciclo da soja, realizaram-se a colheita, a secagem e a análise sanitária das sementes pelo método 'blotter test', referente aos 336 pontos demarcados. Quatro modelos de semivariogramas foram ajustados aos dados coletados utilizando os métodos mínimos quadrados ordinários (OLS), mínimos quadrados ponderados (WLS), máxima verossimilhança (ML) e máxima verossimilhança restrita (REML). A validação cruzada foi empregada para escolha final do modelo e método do semivariograma. Em seguida, efetuaram-se a krigagem e o desvio padrão da krigagem. Os mapas de krigagem ilustraram a transmissão da semente para a semente e a sua variância. Verificou-se estrutura de dependência espacial da transmissão de C. truncatum via semente. O melhor modelo de semivariograma foi o esférico e o melhor ajuste foi o REML. Houve alcance de $0,95,4,03$ e 7,05 m para as parcelas com $0,8,1,6$ e 2,4\% de sementes inoculadas respectivamente. Quanto maior o inóculo primário da parcela, maior foi a transmissão para as sementes próximas à fonte de inóculo.

Palavras-chave adicionais: Máxima verossimilhança restrita, semivariograma esférico, transmissão.

\begin{abstract}
Noetzold, R.; Alves, M.C.; Cassetari Neto, D.; Machado, A.Q.. Spatial variability of Colletotrichum truncatum in soybean field under three seed health levels. Summa Phytopathologica, v.40, n.1, p.16-23, 2014.

This study aimed to characterize the spatial variability of Colletotrichum truncatum (Schwein) incidence among harvested soybean seeds and to determine the best semivariogram model and method to represent $C$. truncatum incidence among these harvested seeds. The experiment was conducted under field conditions in 2009/10 crop cycle, in 3 plots of 9.9 x 10 m, using seeds inoculated with $C$. truncatum. The inoculum corresponded to $0.8,1.6$ and $2.4 \%$ of the total sown seeds. Three meshes were marked with GNSS receivers, totaling 112 points in each plot spaced at $1.3 \mathrm{~m}$ on the row. At the end of soybean cycle, seeds were harvested, dried and analyzed for their health according to the blotter test, considering the 336 demarked points. Four semivariogram models were adjusted

to the collected data by using the methods ordinary least squares (OLS), weighted least squares (WLS), maximum likelihood (ML) and restricted maximum likelihood (REML). Cross-validation was used for the final selection of the semivariogram model and method. Then, kriging and kriging standard deviation were performed. The kriging maps illustrated the seed-to-seed transmission and its variance. Spatial dependence was found for C. truncatum transmission through the seeds. The best semivariogram model was spherical and the best fit was REML. There was a range of 0.95 , 4.03 and $7.05 \mathrm{~m}$ for plots with $0.8,1.6$ and $2.4 \%$ inoculated seeds, respectively. The higher the primary inoculum of the plot, the higher the transmission to seeds near the inoculum source.
\end{abstract}

Additional keywords: Restricted maximum likelihood, spherical semivariogram, transmission.

A geoestatística tem por objetivos caracterizar a variabilidade espacial dos atributos do solo e das plantas e fazer estimativa, utilizando o princípio da variabilidade espacial, a fim de se identificar inter-relações desses atributos no espaço e no tempo, além de permitir estudar padrões de amostragem adequada (24). Na fitopatologia técnicas geoestatísticas estão sendo utilizadas para obter informações de localização e quantificação de doenças $(1,2,19)$.

Alves et al. (2) verificaram aplicação satisfatória da geoestatística 
no estudo do progresso espacial e temporal da antracnose do feijoeiro, tendo em vista o caráter agregado do inóculo e da doença no agroecossistema, detectado por métodos estatísticos clássicos $(21,22)$.

Na soja, o conhecimento da variabilidade espacial de C. truncatum, encontrado nos campos de produção de sementes e de grãos constituise em informações fundamentais para o entendimento do progresso da antracnose e para tomada de decisão de medidas de controle. Caso o inóculo primário, transportado via-semente encontrar ambiente favorável e o hospedeiro for suscetível, podem-se estabelecer focos iniciais de infecção. Posteriormente, o progresso da doença no espaço e no tempo ocorrerá a partir da fonte de inóculo secundário, produzido nas primeiras lesões das plantas infectadas $(6,23)$. Com a queda dos cotilédones e/ou o prostamento de pecíolos com sintomas da antracnose em soja, obtém-se o inóculo secundário. Dependendo das condições climáticas, pode haver o progresso da epidemia na lavoura, ocorrendo assim, a transmissão semente-planta e da planta-semente.

Araújo et al. (4) estudaram a relação entre porcentagens de sementes de algodoeiro infectadas com Colletotrichum gossypii Sout var. cephalosporioides A.S. Costa e o progresso da ramulose no campo e, concluíram que a transmissão do patógeno planta-semente foi maior com aumento do inóculo inicial nas sementes. Porém, com a análise de variância clássica não é possível observar a variabilidade espacial do patógeno, mas ferramentas da geoestatística contribuem na interpretação do padrão espacial do progresso do patógeno.

De acordo com Henning (14), no Brasil não foi estabelecido nível de tolerância para C. truncatum em sementes de soja, do mesmo modo, ainda não se tem muitas informações referentes à transmissibilidade da semente para a planta e da planta para a semente. Nesse sentido, objetivou-se caracterizar a variabilidade espacial da incidência de C. truncatum em sementes colhidas de soja e determinar o melhor modelo e método de semivariograma que represente a incidência de C. truncatum nessas sementes.

\section{MATERIAL E MÉTODOS}

O experimento foi realizado durante o período de agosto de 2009 a abril de 2010, na área experimental localizada em 15³8'38.93" S e $56^{\circ} 05$ ' 58.33 " W e altitude de $180 \mathrm{~m}$ do nível do mar, pertencente ao Centro Universitário de Várzea Grande (UNIVAG), em Várzea Grande, MT.

O isolado de C. truncatum foi obtido de sementes de soja submetidas ao 'blotter test' no Laboratório de Fitopatologia do UNIVAG, safra 2009/2010 e confirmado como tal pelas características biológicas em microscópio ótico (16). O inóculo do patógeno foi multiplicado em meio de BDA. O cultivo foi realizado em placa de Petri de $9 \mathrm{~cm}$ de diâmetro, por 7 dias, sob temperatura de $20{ }^{\circ} \mathrm{C} \pm 2{ }^{\circ} \mathrm{C}$, em regime de $12 \mathrm{~h}$ luz.

Após esse período, 130 sementes de soja (Glicyne max. L.) cultivar TMG 131 RR, suscetível ao fungo C. truncatum e livres desse patógeno, foram distribuídas na placa de Petri contendo a colônia fúngica em crescimento ativo sobre o BDA, dispostas em uma única camada ocupando toda a superfície da placa, por um período de $48 \mathrm{~h}$. Após a inoculação as sementes foram retiradas da placa e colocadas para secar sobre papel toalha em temperatura ambiente por $2 \mathrm{~h}$. As sementes livres do patógeno foram obtidas pelo 'blotter test' modificado com restrição hídrica a -0,6 MPa, com vários lotes do cultivar da soja, sendo que o lote utilizado apresentou 16,5\% de Peniccillium spp.; 16,0\% de Aspergillus spp.; 2,5\% de Fusarium semitectum e 0,5\% de Cercospora kikuchii.

Foram semeadas 4 linhas de milho híbrido (Zea mays L.) cultivar
AG 7088 ao redor das 3 parcelas de soja, distanciadas de $2 \mathrm{~m}$ ao redor das parcelas, com espaçamento entre linhas de $0,50 \mathrm{~m}$ e população de 60.000 plantas ha ${ }^{-1}$.

O solo apresenta textura arenosa e seu preparo foi o convencional (grade aradora e grave niveladora). Após a abertura manual dos sulcos com enxada, o adubo foi distribuído ao lado e abaixo das sementes, conforme recomendação da Embrapa (12). Depois da incorporação do adubo, as sementes foram distribuídas em profundidade de 2 a $3 \mathrm{~cm}$. As sementes não inoculadas com o patógeno foram tratadas, para cada $100 \mathrm{~kg}$ de sementes foram utilizados $300 \mathrm{~mL}$ de tiametoxan $\left(350 \mathrm{gL}^{-1}\right)$; $150 \mathrm{~mL}$ de fipronil $\left(250 \mathrm{gL}^{-1}\right) ; 200 \mathrm{~mL}$ de carbendazim $\left(150 \mathrm{gL}^{-1}\right)$ mais thiran $\left(350 \mathrm{gL}^{-1}\right)$ e $300 \mathrm{~mL}$ de fluquinconazol $\left(167 \mathrm{gL}^{-1}\right)$.

Aos 49 dias após semeadura do milho, as sementes de soja foram semeadas nas 3 parcelas, ambas distanciadas a $2 \mathrm{~m}$ das linhas de milho, cada uma contendo 20 linhas de $10 \mathrm{~m}$ de comprimento espaçadas de $0,45 \mathrm{~m}$, sendo consideradas úteis as 16 linhas centrais. Foram semeadas entre 16,5 a 20 sementes de soja por metro linear no sentido norte-sul. Em cada parcela experimental foram semeadas quantidades de sementes inoculadas com C. truncatum, para obtenção de níveis iniciais do patógeno com $0,8 \% ; 1,6 \%$ e 2,4\% de incidência. Assim, 20 sementes foram semeadas em 1 linha da parcela com $0,8 \%$ de incidência, 40 sementes foram distribuídas em 2 linhas da parcela com 1,6\% de incidência e 60 sementes foram semeadas em 3 linhas da parcela com $2,4 \%$ de incidência. Todas as sementes foram distribuídas no centro de cada parcela, em que cada linha correspondeu a $1 \mathrm{~m}$ linear (Figura 1).

Utilizou-se um par de receptores Global Navigation Satellite Systems (GNSS) configurado em datum WGS 84, zona UTM $21 \mathrm{~K}$, modo real time kinematic (RTK), para demarcar em cada parcela uma grade com 112 pontos amostrais correspondendo as 16 linhas centrais de semeadura (Figura 1).

A colheita manual foi realizada em cada ponto amostral e em seguida efetuaram-se a debulha e a secagem das sementes, as quais foram homogeneizadas e submetidas ao 'blotter test' modificado com restrição hídrica com manitol a - 0,6 MPa e ágar a 0,2\% (17). Foram distribuídas 25 sementes por placa, para cada ponto amostral. Essas placas de Petri com as sementes permaneceram na sala de crescimento com temperatura de $22{ }^{\circ} \mathrm{C} \pm 2{ }^{\circ} \mathrm{C}$, e fotoperíodo de $12 \mathrm{~h}$. A leitura de incidência de $C$. truncatum nas sementes colhidas foi realizada aos 7 dias após a incubação, com auxílio de microscópio estereoscópio, sendo verificado a presença ou ausência de $C$. truncatum em cada semente.

A partir da análise exploratória dos dados, a geoestatística foi utilizada para estudar a variabilidade espacial de C. truncatum, bem como para escolher o modelo de semivariograma e método de ajuste que descreveu melhor a variabilidade dos dados.

A dependência espacial da transmissibilidade de C. truncatum nas sementes foi analisada por meio de ajustes de semivariogramas, com base na pressuposição de estacionariedade da hipótese intrínseca. O semivariograma experimental foi estimado de acordo com o estimador robusto (9).

$$
\gamma(u)=\left[\frac{1}{N_{u}} \sum \frac{N_{u}}{i=1}\left\{S\left(x_{i}+u\right)-S\left(x_{i}\right)\right\}^{1 / 2}\right]^{4} / 0,914+\frac{0,988}{N_{u}}
$$

em que $\gamma(u)$ corresponde a semivariância estimada e aos números de pares de valores das observações $\quad S\left(x_{i}+u\right)-S($ sepa-rados pela distância $u$. A transformação da raiz quadrada das diferenças é apresentada como tendo momentos parecidos com aqueles da distribuição normal e o denominador da equação foi utilizado para corrigir tendências nos dados.

Os modelos teóricos esférico, exponencial, gaussiano e matérn de 

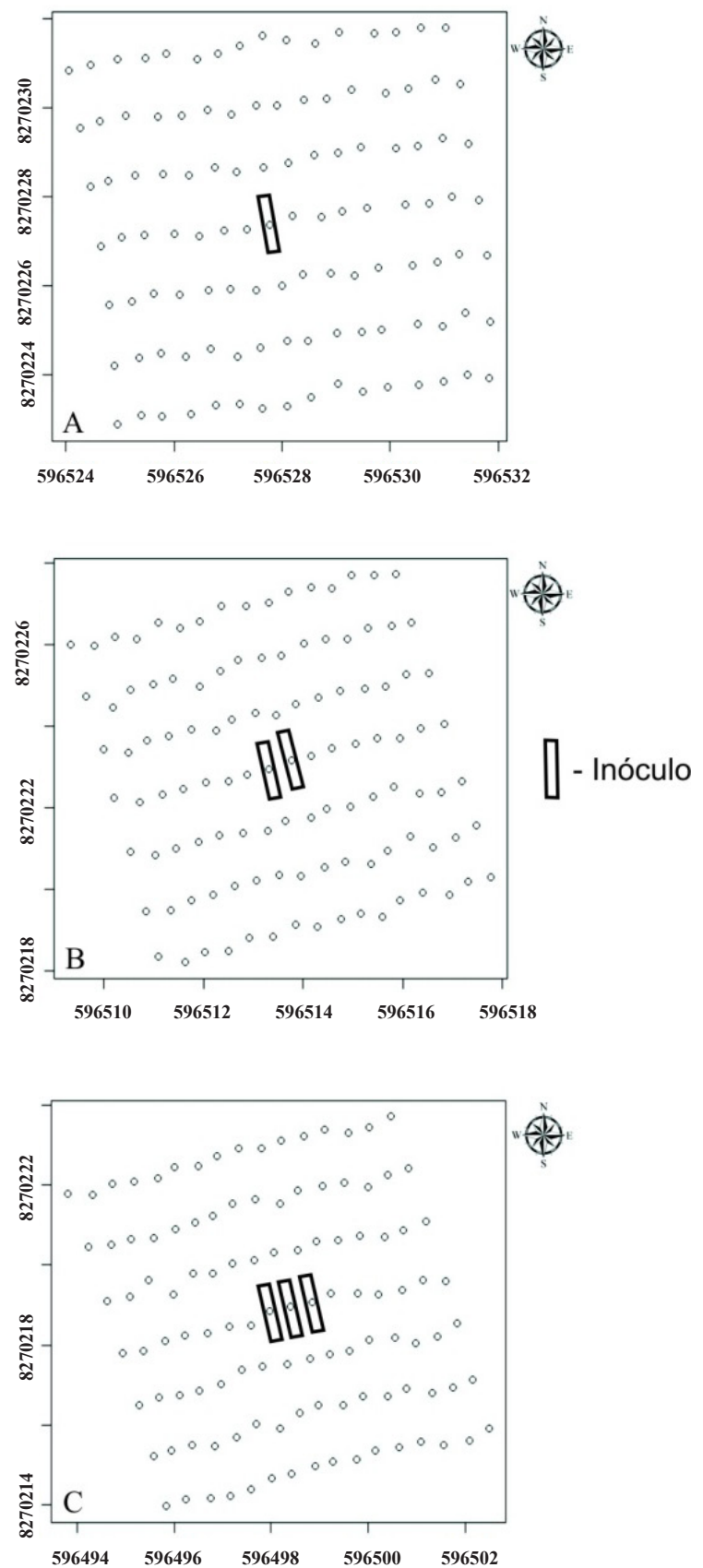

Figura 1. Malhas amostrais realizadas nas parcelas com $0,8 \%$ (A), 1,6\% (B) e 2,4\% (C) de sementes inoculadas com Colletotrichum truncatum.

semivariogramas isotrópicos utilizados foram definidos de acordo com Diggle e Ribeiro Junior (9).

Modelo esférico: $\rho(u)=\left\{1-\frac{3}{2}(u / \phi)+\frac{1}{2}(u / \phi)^{3} \begin{array}{c}: 0 \leq u \leq \phi \\ 0: u>\phi\end{array}\right.$

Modelo exponencial: $\rho(u)=\exp \left\{-(u / \phi)^{k}\right\}$

Modelo gaussiano: $\rho(u)=\exp \left\{-\left(\phi^{2} / u^{2}\right)\right\}$

Modelo matérn: $\rho(u)=\left\{2^{k-1} \Gamma(k)\right\}^{-1}(u / \phi)^{k} k_{k}(u / \phi)$

Em que $k=1$ em que $\phi$ é o alcance e, $u$ é à distância.

Com o cálculo das semivariâncias, foram ajustados aos semivariogramas experimentais, os modelos testados pelo método OLS e WLS. Os semivariogramas também foram ajustados aos dados pelo ML e REML. Sequencialmente, foi realizada a interpolação por krigagem ordinária para mapear a variabilidade espacial de $C$. truncatum em sementes de soja, conforme Diggle e Ribeiro Junior (9).

$$
\hat{S}(x)=\sum a_{i}(x) Y_{i}
$$

em que $S(x)$ é o estimador da krigagem ordinária, $a_{i}(x)$ são os pesos da predição ou pesos de krigagem, e possuem a propriedade de que $\sum a_{i}(x)=1$ para qualquer localização de $x$, e $Y_{i}$ são os dados observados.

O método da validação cruzada foi empregado para escolha do modelo de semivariograma teórico (8). Em seguida efetuou-se a comparação entre os modelos, em cada método de ajuste, por meio dos coeficientes de qualidade do erro médio (ME) e quadrado médio do desvio padrão (MSDR).

$$
\begin{gathered}
\mathrm{ME}=\frac{1}{N} \sum_{i=1}^{N}\left\{S\left(x_{i}\right)-\hat{S}\left(x_{i}\right)\right\} \\
\mathrm{MSDR}=\frac{1}{N} \sum_{i=1}^{N} \sqrt{\frac{\left\{S\left(x_{i}\right)-\hat{S}\left(x_{i}\right)\right\}^{2}}{\sigma^{2}\left(x_{i}\right)}}
\end{gathered}
$$

em que $S\left(x_{i}\right)$ é o valor observado no ponto $\left(x_{i}\right) ; S\left(x_{i}\right)$ é o valor estimado pela krigagem para o ponto $\left(x_{i}\right)$ e $\sigma\left(x_{i}\right)$ é a variância da krigagem.

Para o modelo do variograma ser considerado satisfatório, o valor do MSDR deve ser próximo ou igual a 1 (25), e o valor de EM próximo ou igual a 0 (13). Após a escolha do melhor método e modelo de ajuste, efetuou-se o mapeamento do desvio padrão da krigagem de cada inóculo. Todas as análises geoestatísticas foram realizadas no 'software' R com o pacote geoR (9).

\section{RESULTADOS E DISCUSSÃO}

O método de inoculação do fungo foi eficiente, sendo que germinaram $57,5 \% ; 43,75 \%$ e $48,33 \%$ plântulas sintomáticas, referente às sementes de soja inoculadas com $0,8 \%, 1,6 \%$ e $2,4 \%$ de C. truncatum, respectivamente. Todas as sementes germinadas apresentaram os primeiros sintomas nos cotilédones com manchas deprimidas de coloração castanho-escuras. Depois da queda dos cotilédones, as plantas não apresentaram sintomas ou sinais da doença e do patógeno até o final do florescimento. Após esse período observou-se sintomas em folhas, hastes e posteriormente nas vagens.

Com base na análise exploratória não espacial, observou-se que quanto maior o inóculo primário, menor foram os valores de assimetria e de coeficiente de variação. O elevado valor do coeficiente de variação entre 64,78 e $91 \%$, foi justificado pelos diferentes valores encontrados nos pontos amostrais. Os dados da parcela com maior inóculo apresentaram maior média, valor máximo e desvio padrão (Tabela 1).

A distribuição espacial e a expansão de Huanglongbing em citrus foi avaliada por Leal et al. (15) e, também observaram elevados valores de coeficiente de variação sendo que o maior valor foi $313,05 \%$, 
Tabela 1. Estatística descritiva referente às parcelas com 0,$8 ; 1,6$ e 2,4\% de sementes de soja inoculadas com Colletotrichum truncatum.

\begin{tabular}{cccc}
\hline Índices & \multicolumn{3}{c}{ Sementes inoculadas (\%) } \\
\cline { 2 - 4 } Estatísticos & 0,8 & 1,6 & 2,4 \\
\hline Curtose & 0,50 & 0,69 & $-0,89$ \\
Assimetria & 1,05 & 0,85 & 0,16 \\
Mínimo & 0,00 & 0,00 & 0,00 \\
Média & 17,29 & 11,68 & 35,12 \\
Mediana & 12,00 & 12,00 & 36,00 \\
Máximo & 64,00 & 44,00 & 92,00 \\
Desvio padrão & 15,73 & 9,90 & 22,75 \\
Coefíciente de variação (\%) & 91,00 & 84,79 & 64,78 \\
\hline
\end{tabular}

correspondente ao talhão com a menor média do número de plantas com greening dos 46 talhões de citros avaliados.

Para quantificar a estrutura e magnitude de dependência espacial entre as amostras em estudo, utilizou-se a geoestatística. Para avaliar a variabilidade espacial do fungo nas sementes, e por meio de ajustes de semivariogramas foi possível quantificar a magnitude e a estrutura de dependência espacial da transmissão semente-semente com $C$. truncatum nos diferentes níveis de inóculo inicial testados.

Todas as combinações testadas entre método e modelo de ajuste de semivariograma resultaram em dependência espacial (Tabela 2). O modelo isotrópico esférico ajustado ao método REML apresentou os melhores coeficientes de qualidade obtidos pela validação cruzada, para os níveis de inóculo inicial testado (Tabela 3), sendo utilizado para a representação variográfica e para a confecção dos mapas de krigagem (Figura 2). Observou-se que quanto maior o inóculo inicial

Tabela 2. Parâmetros e tipos de modelos de semivariogramas referente às parcelas com 0,$8 ; 1,6$ e 2,4\% de sementes de soja inoculadas com Colletotrichum truncatum.

\begin{tabular}{|c|c|c|c|c|c|c|}
\hline $\begin{array}{c}\text { Sementes } \\
\text { inoculadas (\%) }\end{array}$ & Modelo & Método & Efeito pepita & Patamar & $\begin{array}{c}\text { Alcance } \\
\text { (m) }\end{array}$ & $\begin{array}{c}\text { Alcance prático } \\
\text { (m) }\end{array}$ \\
\hline 0,8 & esférico & OLS & 65,53 & 180,88 & 1,36 & 1,36 \\
\hline 0,8 & exponencial & OLS & 85,32 & 194,83 & 1,22 & 3,66 \\
\hline 0,8 & gaussiano & OLS & 0,00 & 175,97 & 0,23 & 0,39 \\
\hline 0,8 & matérn & OLS & 98,05 & 191,68 & 0,78 & 3,13 \\
\hline 0,8 & esférico & WLS & 119,76 & 195,13 & 4,62 & 4,62 \\
\hline 0,8 & exponencial & WLS & 106,78 & 208,53 & 2,23 & 6,67 \\
\hline 0,8 & gaussiano & WLS & 134,01 & 196,70 & 2,41 & 4,18 \\
\hline 0,8 & matérn & WLS & 121,84 & 203,91 & 1,44 & 5,77 \\
\hline 0,8 & esférico & ML & 182,70 & 281,04 & 11,28 & 11,28 \\
\hline 0,8 & exponencial & ML & 178,70 & 286,00 & 5,63 & 16,87 \\
\hline 0,8 & gaussiano & ML & 196,50 & 327,50 & 7,33 & 12,69 \\
\hline 0,8 & matérn & ML & 189,20 & 302,20 & 4,11 & 16,43 \\
\hline 0,8 & esférico & REML & 74,21 & 248,41 & 0,95 & 0,95 \\
\hline 0,8 & exponencial & REML & 71,84 & 251,84 & 0,66 & 1,96 \\
\hline 0,8 & gaussiano & REML & 142,23 & 296,75 & 6,52 & 11,29 \\
\hline 0,8 & matérn & REML & 192,20 & 6066,20 & 51,89 & 207,50 \\
\hline 1,6 & esférico & OLS & 0,00 & 126,34 & 4,41 & 4,41 \\
\hline 1,6 & exponencial & OLS & 0,00 & 201,60 & 4,01 & 12,02 \\
\hline 1,6 & gaussiano & OLS & 22,22 & 131,86 & 2,37 & 4,09 \\
\hline 1,6 & matérn & OLS & 10,67 & 156,27 & 1,69 & 6,74 \\
\hline 1,6 & esférico & WLS & 0,00 & 125,69 & 4,29 & 4,29 \\
\hline 1,6 & exponencial & WLS & 0,00 & 191,46 & 3,67 & 10,98 \\
\hline 1,6 & gaussiano & WLS & 18,66 & 130,60 & 2,26 & 3,91 \\
\hline 1,6 & matérn & WLS & 3,71 & 150,50 & 1,50 & 6,00 \\
\hline 1,6 & esférico & ML & 17,84 & 110,89 & 4,00 & 4,00 \\
\hline 1,6 & exponencial & ML & 7,69 & 98,09 & 1,35 & 4,03 \\
\hline 1,6 & gaussiano & ML & 36,49 & 103,81 & 2,05 & 3,55 \\
\hline 1,6 & matérn & ML & 20,72 & 97,52 & 0,91 & 3,64 \\
\hline 1,6 & esférico & REML & 17,12 & 115,25 & 4,03 & 4,03 \\
\hline 1,6 & exponencial & REML & 9,38 & 109,88 & 1,64 & 4,92 \\
\hline 1,6 & gaussiano & REML & 36,70 & 114,52 & 2,18 & 3,77 \\
\hline 1,6 & matérn & REML & 21,83 & 106,36 & 1,03 & 4,11 \\
\hline
\end{tabular}

continua.. 
Tabela 2. Parâmetros e tipos de modelos de semivariogramas referente às parcelas com 0,8; 1,6 e 2,4\% de sementes de soja inoculadas com Colletotrichum truncatum.

\begin{tabular}{ccccccc}
\hline $\begin{array}{c}\text { Sementes } \\
\text { inoculadas (\%) }\end{array}$ & Modelo & Método & Efeito pepita & Patamar & $\begin{array}{c}\text { Alcance } \\
(\mathbf{m})\end{array}$ & $\begin{array}{c}\text { Alcance prático } \\
(\mathbf{m})\end{array}$ \\
\hline 2,4 & esférico & OLS & 148,61 & 767,22 & 8,83 & 8,83 \\
2,4 & exponencial & OLS & 113,64 & 932,24 & 5,34 & 15,99 \\
2,4 & gaussiano & OLS & 207,07 & 819,64 & 3,99 & 6,90 \\
2,4 & matérn & OLS & 169,04 & 836,68 & 2,75 & 10,98 \\
2,4 & esférico & WLS & 86,76 & 717,13 & 6,98 & 6,98 \\
2,4 & exponencial & WLS & 41,82 & 871,22 & 4,07 & 12,18 \\
2,4 & gaussiano & WLS & 194,10 & 730,41 & 3,62 & 6,26 \\
2,4 & matérn & WLS & 128,46 & 806,05 & 2,35 & 9,41 \\
2,4 & esférico & ML & 204,60 & 459,40 & 5,85 & 5,85 \\
2,4 & exponencial & ML & 198,80 & 546,10 & 4,30 & 12,87 \\
2,4 & gaussiano & ML & 231,00 & 492,93 & 2,80 & 4,84 \\
2,4 & matérn & ML & 220,50 & 543,60 & 2,37 & 9,47 \\
2,4 & esférico & REML & 204,20 & 517,10 & 7,05 & 7,05 \\
2,4 & exponencial & REML & 202,40 & 967,90 & 10,62 & 31,83 \\
2,4 & gaussiano & REML & 231,00 & 533,30 & 2,93 & 5,08 \\
2,4 & matérn & REML & 222,50 & 694,90 & 3,11 & 12,44 \\
\hline
\end{tabular}

Tabela 3. Validação cruzada da krigagem referente às parcelas com 0,$8 ; 1,6 \mathrm{e}$ 2,4\% de sementes de soja inoculadas com Colletotrichum truncatum.

\begin{tabular}{|c|c|c|c|c|}
\hline $\begin{array}{c}\text { Sementes } \\
\text { inoculadas (\%) }\end{array}$ & Modelo & Método & $\mathrm{ME}$ & MSDR \\
\hline 0,8 & esférico & OLS & 0,05 & 1,53 \\
\hline 0,8 & exponencial & OLS & 0,01 & 1,49 \\
\hline 0,8 & gaussiano & OLS & 0,01 & 1,39 \\
\hline 0,8 & matérn & OLS & 0,01 & 1,52 \\
\hline 0,8 & esférico & WLS & 0,00 & 1,41 \\
\hline 0,8 & exponencial & WLS & 0,01 & 1,44 \\
\hline 0,8 & gaussiano & WLS & 0,00 & 1,41 \\
\hline 0,8 & matérn & WLS & 0,00 & 1,42 \\
\hline 0,8 & esférico & ML & 0,00 & 1,01 \\
\hline 0,8 & exponencial & ML & 0,00 & 1,01 \\
\hline 0,8 & gaussiano & ML & $-0,01$ & 1,00 \\
\hline 0,8 & Matérn & ML & 0,00 & 1,01 \\
\hline 0,8 & esférico & REML & 0,05 & 1,02 \\
\hline 0,8 & exponencial & REML & 0,03 & 1,10 \\
\hline 0,8 & gaussiano & REML & 0,01 & 1,37 \\
\hline 0,8 & matérn & REML & 0,00 & 1,00 \\
\hline 1,6 & esférico & OLS & 0,02 & 1,90 \\
\hline 1,6 & exponencial & OLS & 0,02 & 1,67 \\
\hline 1,6 & gaussiano & OLS & 0,00 & 1,56 \\
\hline 1,6 & matérn & OLS & 0,01 & 1,72 \\
\hline 1,6 & esférico & WLS & 0,02 & 1,86 \\
\hline 1,6 & exponencial & WLS & 0,02 & 1,61 \\
\hline 1,6 & gaussiano & WLS & $-0,01$ & 1,83 \\
\hline 1,6 & matérn & WLS & 0,04 & 2,69 \\
\hline 1,6 & esférico & ML & $-0,01$ & 0,98 \\
\hline
\end{tabular}

continua...
Tabela 3. Validação cruzada da krigagem referente às parcelas com 0,$8 ; 1,6 \mathrm{e}$ $2,4 \%$ de sementes de soja inoculadas com Colletotrichum truncatum.

\begin{tabular}{|c|c|c|c|c|}
\hline $\begin{array}{c}\text { Sementes } \\
\text { inoculadas (\%) }\end{array}$ & Modelo & Método & $\mathrm{ME}$ & MSDR \\
\hline 1,6 & exponencial & ML & $-0,01$ & 0,98 \\
\hline 1,6 & gaussiano & ML & $-0,01$ & 0,98 \\
\hline 1,6 & matérn & ML & $-0,01$ & 0,98 \\
\hline 1,6 & esférico & REML & 0,00 & 0,98 \\
\hline 1,6 & exponencial & REML & 0,00 & 0,98 \\
\hline 1,6 & gaussiano & REML & $-0,01$ & 0,98 \\
\hline 1,6 & matérn & REML & 0,00 & 0,98 \\
\hline 2,4 & esférico & OLS & $-0,03$ & 1,19 \\
\hline 2,4 & exponencial & OLS & $-0,05$ & 1,33 \\
\hline 2,4 & gaussiano & OLS & $-0,05$ & 2,44 \\
\hline 2,4 & matérn & OLS & $-0,03$ & 0,99 \\
\hline 2,4 & esférico & WLS & $-0,04$ & 1,66 \\
\hline 2,4 & exponencial & WLS & $-0,06$ & 2,11 \\
\hline 2,4 & gaussiano & WLS & $-0,03$ & 1,19 \\
\hline 2,4 & matérn & WLS & $-0,04$ & 1,54 \\
\hline 2,4 & esférico & ML & $-0,04$ & 0,99 \\
\hline 2,4 & exponencial & ML & $-0,04$ & 0,99 \\
\hline 2,4 & gaussiano & ML & $-0,04$ & 0,99 \\
\hline 2,4 & matérn & ML & $-0,03$ & 0,99 \\
\hline 2,4 & esférico & REML & $-0,03$ & 0,99 \\
\hline 2,4 & exponencial & REML & $-0,04$ & 0,99 \\
\hline 2,4 & gaussiano & REML & $-0,04$ & 0,99 \\
\hline 2,4 & matérn & REML & $-0,04$ & 1,22 \\
\hline
\end{tabular}

ME $=$ erro médio

MSDR = quadrado médio do desvio padrão 
de sementes de soja com C. truncatum, maior foi o alcance (Tabela 2) e, quanto maior o inóculo primário da parcela, maior foi a transmissão da semente para semente próxima à fonte de inóculo (Figura 2).

Com base nas Tabelas 2 e 3 notaram-se outras combinações de ajuste entre modelo e método com coeficientes de qualidades iguais ou muito próximos dos encontrados no modelo e método escolhido, porém, na maioria desses casos o valor correspondente do alcance ultrapassou a distância real das parcelas experimentais. Alves et al. (3) caracterizaram a estrutura espacial de pragas do cafeeiro e, obtiveram também melhor aplicação do semivariograma esférico ajustado pelo método REML.
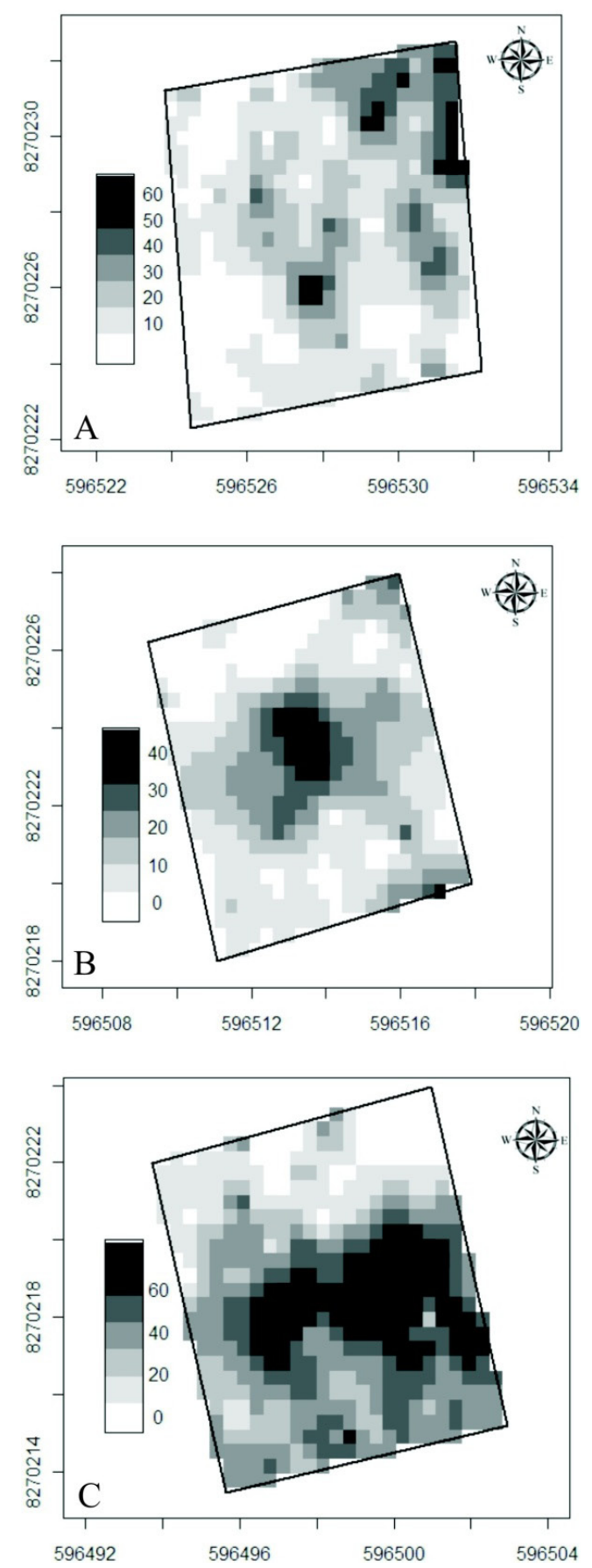

Figura 2. Mapas de krigagem da incidência de Colletotrichum truncatum nas sementes referente às parcelas com $0,8 \%(\mathrm{~A}), 1,6 \%(\mathrm{~B})$ e $2,4 \%(\mathrm{C})$ de sementes inoculadas com o fungo.
Charest et al. (7), estudando a distribuição espacial de Venturia inaequalis (Cooke) G. Winter, e Dinardo-Miranda e Fracasso (10) pesquisando a distribuição espacial de nematoides, observaram melhor ajuste com o semivariograma esférico. Dinardo-Miranda et al. (11) analisaram a dependência espacial de Mahanarva fimbriolata (Stål) somente após o início da segunda geração dessa praga e observaram, melhor aplicação do semivariograma esférico. Outros pesquisadores $(5,20)$, também relataram que o semivariograma esférico, foi o que melhor descreveu os atributos de solo e de planta nas áreas estudadas. Mouen Bedimo et al. (18) pesquisaram em duas áreas a dinâmica espaço-temporal da antracnose dos frutos verdes do cafeeiro causada por Colletotrichum kahawae e verificaram ajuste de semivariograma esférico e gaussiano.

Por meio dos mapas de krigagem observou-se que quanto maior o inóculo primário, maior o padrão de distribuição agregada da transmissão semente para semente (Figura 2). Somente na parcela com $0,8 \%$ de sementes inoculadas, a transmissão de $C$. truncatum apresentou maior ocorrência no centro da parcela e no lado nordeste.

No nível de $0,8 \%$ de inóculo, focos secundários surgiram nessa parcela, o que resultou em maior valor de semivariância e menor valor de alcance (Tabela 2). A justificativa provável da ocorrência do patógeno apresentar foco secundário, na região nordeste da parcela (Figura 2), pode ser explicada pela maior população de plantas de soja nessa área aliada ao sentido da direção do sistema de irrigação por pivô central.

Na parcela com $0,8 \%$ de sementes inoculadas houve aumento na distância entre os focos secundários (Figura 2). Resultados semelhantes foram encontrados por Alves et al. (2), que avaliando a severidade da antracnose em plantas de feijoeiro, constataram pela krigagem, formação de focos secundários além do foco primário, o que indica ocorrência de dispersão de inóculo e ciclos secundários da doença. Essa capacidade de dispersar pode ser uma característica da sobrevivência do C. truncatum. Talamini (22) afirmou que o progresso da antracnose pode ser rápido se o ambiente for favorável à epidemia.

Na parcela com o menor inóculo inicial o padrão foi de forma geral agregado aleatório (Figura 2), pois além do inóculo inicial houve formação de focos secundários apresentando maior variação do desvio padrão da krigagem (Figura 3). No inóculo com 1,6\% de C. truncatum nas sementes, o padrão da incidência desse patógeno foi agregado e resultou em menor variação do desvio padrão da krigagem (Figura 3). Para a parcela com 2,4\% de sementes inoculadas com C. truncatum, esse padrão foi considerado também agregado, havendo infecção de quase todas as plantas da parcela, sendo verificada ausência desse fungo nas sementes em apenas $8,93 \%$ dos pontos amostrais. Contudo, as maiores incidências ocorreram no inóculo inicial e ao seu redor (Figura 2).

A parcela com $1,6 \%$ de sementes inoculadas teve maior transmissão agrupada no centro da parcela e poucos pontos espalhados aleatoriamente nas proximidades do foco primário. A parcela com $2,4 \%$ de sementes inoculadas apresentou maior transmissão no centro da parcela, espalhando-se mais para o sentido sudeste, até a bordadura da parcela (Figura 2). Resultado semelhante foi encontrado por Alves et al. (2), ao caracterizar o progresso espaço-temporal da severidade da antracnose do feijoeiro e da ramulose do algodoeiro.

Musoli et al. (19) estudaram por meio de técnicas geoestatísticas a estrutura espaço-temporal da murcha do café causada por Fusarium xylarioides Steyaert (Gibberella xylarioides Heim e Saccas) em Coffea canephora e, observaram progresso temporal lento e padrão espacial agregado. Almeida et al. (1), em estudo sobre a dependência espacial de Phytophthora nicotianae Breda de Haan (1896), com ajuste de semivariograma isotrópico esférico, detectaram ausência de correlação espacial do patógeno entre as amostras avaliadas. 

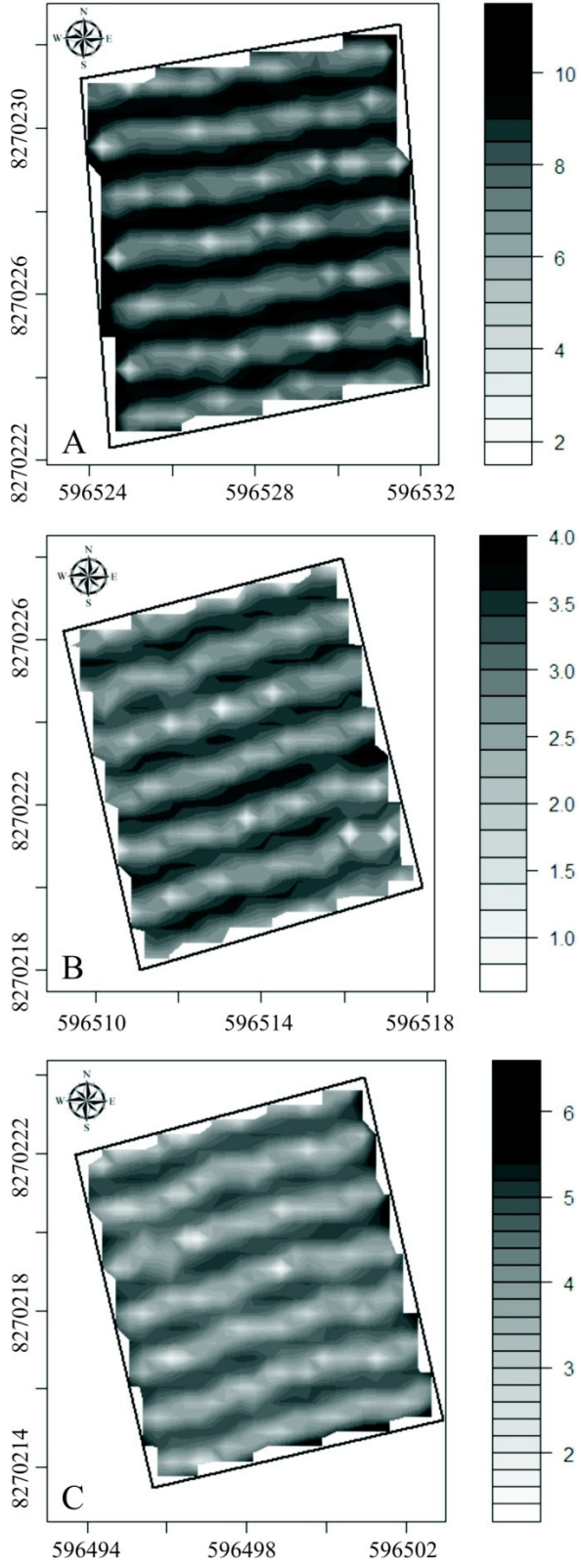

Figura 3. Mapas do desvio padrão da krigagem da incidência de Colletotrichum truncatum nas sementes referente às parcelas com $0,8 \%$ (A), 1,6\% (B) e 2,4\% (C) de sementes inoculadas com o fungo.

Araújo et al. (4) observaram que a área abaixo da curva de progresso da incidência, da severidade da ramulose, e a transmissão do C. gossypii var. cephalosporioides planta-semente foram maiores com aumento do inóculo inicial nas sementes.

Os maiores erros da krigagem foram localizados mais distantes dos pontos amostrados e, a maior variância foi constatada na parcela com $0,8 \%$ de sementes inoculadas com C. truncatum (acima de $10 \%$ de incidência do patógeno nas sementes). Provavelmente isso ocorreu por esta parcela apresentar maior incerteza da predição da krigagem (Figura 3).

Neste trabalhou verificou-se a tendência de maior transmissão semente-semente de C. truncatum e menor desvio padrão da krigagem (Figura 3) quando observou-se padrão de agregação, com maior centralização ao redor do inóculo inicial da parcela com 1,6\% de sementes de soja inoculadas com o patógeno.

O conhecimento do padrão espacial do $C$. truncatum em sementes de soja é uma ferramenta essencial para estabelecer táticas e estratégias precoces de controle. $\mathrm{O}$ fungo pode ficar em latência após a queda dos cotilédones e dependendo das condições edafoclimáticas e da planta, a latência pode permanecer por praticamente todo o ciclo da soja, consequentemente dificultando a identificação dos sintomas e a adoção de medidas preventivas de controle. Quanto aos níveis de tolerância de C. truncatum nas sementes de soja, este certamente será muito abaixo do nível mínimo de incidência do patógeno, estudado neste trabalho $(0,8 \%)$, tendo em vista os altos valores de infecção encontrados ao final do ciclo.

\section{REFERÊNCIAS BIBLIOGRÁFICAS}

1. Almeida, F. A.; Barreto, M.; Barbosa, J. C.; Costa, F. M. Distribuição espacial de Phytophthora nicotianae e reação de cultivares de cebola ao fungo. Summa Phytopathologica, Botucatu, v. 37, n. 1, p. 13-17, 2011.

2. Alves, M. C.; Pozza, E. A.; Machado, J. C.; Araújo, D. V.; Talamini, V.; Oliveira, M. S. Geoestatística como metodologia para estudar a dinâmica espaço-temporal de doenças associadas a Colletotrichum spp. transmitidos por sementes. Fitopatologia Brasileira, Brasília, DF, v. 31, n. 6, p. 557-563, 2006.

3. Alves, M. C.; Silva, F. M.; Moraes, J. C.; Pozza, E. A.; Oliveira, M. S.; Souza, J. C. S.; Alves, L. S. Geostatistical analysis of the spatial variation of the berry borer and leaf miner in a coffee agroecosystem. Precision Agriculture, Dordrecht, v. 12, p. 18-31, 2011.

4. Araújo, D. V.; Zambenedetti, E. B.; Machado, J. C.; Celano, F. A. O. Relação entre níveis de inóculo de Colletotrichum gossypii var. cephalosporioides nas sementes e o progresso da ramulose do algodoeiro. Fitopatologia Brasileira, Brasília, DF, v. 31, n. 2, p. 147-151, 2006.

5. Cambardella, C. A.; Moorman, T. B.; Novak, J. M.; Parkin, T. B.; Karlen, D. L.; Turco, R. F.; Konopka, A. E. Field-scale variability of soil properties in Central Iowa soils. Soil Science Society of America Journal, Madison, v. 58, p. 1501-1511, 1994.

6. Campbell, C. L.; Madden, L. V. Introduction to plant disease epidemiology. New York: J. Wiley, 1990. 532p.

7. Charest, J.; Dewdney, M.; Paulitz, T.; Philion, V.; Carisse, O. Spatia distribution of Venturia inaequalis airborne ascospores in Orchards. Phytopathology, St. Paul, v. 92, p. 769-779, 2002.

8. Cressie, A. G. Statistics for spatial data. New York: Wiley, 1993. 900p.

9. Diggle, P. J.; Ribeiro Junior, P. J. Model-based geoestatistics. New York: Springer, 2007. 228p.

10. Dinardo-Miranda, L. L.; Fracasso, J. V. Spatial distribution of plant-parasitic nematodes in sugarcane fields. Scientia Agricola, Piracicaba, v. 66, n. 2, p. 188-194, 2009.

11. Dinardo-Miranda L. L.; Vasconcelos, A. C. M.; Vieira, S. R.; Fracasso, J. V.; Grego, C. R. Uso da geoestatística na avaliação da distribuição espacial de Mahanarva fimbriolata em cana-de-açúcar. Bragantia, Campinas, v. 66, n. 3, p. 449-455, 2007.

12. Embrapa. Tecnologias de Produção de Soja - Região Central do Brasil, 2009/2010. Londrina: Embrapa Soja; Embrapa Cerrados; Embrapa Agropecuária Oeste, 2008. 262p.

13. Faraco, M. A.; Uribe-Opazo, M. A.; Silva, E. A. A.; Johann, J. A.; Borssoi, J. A. Seleção de modelos de variabilidade espacial para elaboração de mapas temáticos de atributos físicos do solo e produtividade da soja. Revista Brasileira de Ciência do Solo, Viçosa, MG, v. 32, n. 2, p.463-476, 2008.

14. Henning, A. A. Patologia e tratamento de sementes: noções gerais. Documentos Embrapa Soja, n. 264, Londrina, 2005. 52p. Disponível em: <http://www.cnpso.embrapa.br/download/alerta/documento264.pdf $>$. Acesso em: 28 maio 2013.

15. Leal, R. M.; Barbosa, J. C.; Costa, M. G.; Belasque Junior, J.; Yamamoto, P. T.; Dragone, J. Distribuição espacial de Huanglongbing (Greening) em citros utilizando geoestatística. Revista Brasileira de Fruticultura, Jaboticabal, v. 32, n. 3, p. 1-8, 2010.

16. Luz, W. V. (Ed.) Micologia avançada: taxonomia de fungos anamórficos II. Coelomicetos. Passo Fundo: RAPP, 2012. v. III B, 400p.

17. Machado, J. C.; Langerak, C. J. General incubation methods for routine 
seed health analysis. In: Machado J. C.; Langerak C. J.; Jaccoud-Filho, D. S. Seed-borne fungi: a contribution to routine seed health analysis. Bassersdorf, ISTA, 2002. p. 48-80.

18. Mouen Bedimo, J. A.; Bieysse, D.; Cilas, C.; Nottéghem J. L. Spatio-temporal dynamics of arabica coffee berry disease caused by Colletotrichum kahawae on a plot scale. Plant Disease, St. Paul, v. 91, n. 10, p. 12291236, 2007.

19. Musoli, C. P.; Pinard, F.; Charrier, A.; Kangire, A.; Hoopen, G. M.; Kabole, C.; Ogwang, J.; Bieysse, D.; Cilas, C. Spatial and temporal analysis of coffee wilt disease caused by Fusarium xylarioides in Coffea canephora. European Journal of Plant Pathology, New York, v. 122, p. 451-460, 2008.

20. Paz, A.; Taboada, M. T.; Gómez, M. J. Spatial variability in topsoil micronutrients contents in one-hectare cropland plot. Communication in Soil Science and Plant Analysis, New York, v. 27, p. 479-503, 1996.

21. Pinto, A. C. S.; Pozza, E. A.; Talamini, V.; Machado, J. C.; Sales, N. L. P.; Garcia Júnior, D.; Santos, D. M. Análise do padrão espacial e do gradiente da antracnose do feijoeiro em duas épocas de cultivo. Summa Phytopathologica, Jaboticabal, v. 27, n. 4, p. 392-398, 2001.

22. Talamini, V.; Pozza, E. A.; Machado, J. C.; Oliveira, F. A. Epidemiologia de doenças associadas a Colletotrichum spp. transmitidas por sementes. Revisão Anual de Patologia de Plantas, Passo Fundo, v. 10, p. 219 248, 2002.

23. Tanaka, M. A. S.; Menten, J. O. M. Relação entre a resistência do algodoeiro à ramulose e a transmissão de Colletotrichum gossypii var. cephalosporiodes pelas sementes. Summa Phytopathologica, Piracicaba, v. 18, p. 227-234, 1992.

24. Vieira, S. R. Geoestatística em estudos de variabilidade espacial do solo. In: Novais, R.F. de; Alvarez, V. V. H.; Schaefer, C. E. G. R. (Ed.). Tópicos em ciência do solo. Viçosa, MG: Sociedade Brasileira de Ciência do Solo, 2000. v. 1, p. 1-54

25. Webster, R.; Oliver, M. A. Sample adequately to estimate variograms of soil properties. Journal of Soil Science, Philadelphia, v. 43, n. 1, p. 177 192. 2007. 